\title{
Vacuum-Assisted Closure: A Novel Method of Managing Surgical Necrotizing Enterocolitis
}

\author{
Stephanie Sea ${ }^{1}$ Teerin Meckmongkol ${ }^{1}$ Matthew L. Moront ${ }^{1}$ Shaheen Timmapuri ${ }^{1}$ Rajeev Prasad ${ }^{1}$ \\ Marshall Z. Schwartz ${ }^{1} \quad$ L. Grier Arthur ${ }^{1}$ \\ ${ }^{1}$ Department of Pediatric Surgery, St. Christopher's Hospital for \\ Children, Philadelphia, Pennsylvania, United States \\ Eur J Pediatr Surg 2015;25:41-45. \\ Address for correspondence Stephanie Sea, MD, Department of \\ Pediatric Surgery, St. Christopher's Hospital for Children, 3601 A St \\ Philadelphia, PA 19134, United States (e-mail: stephanieasea@gmail.com; \\ Grier.Arthur@tenethealth.com).
}

\begin{abstract}
Keywords

- necrotizing enterocolitis

- abdominal compartment syndrome

- vacuum-assisted closure

Purpose Necrotizing enterocolitis (NEC) requiring surgical intervention is associated with mortality rates approaching $50 \%$. We evaluated outcomes of patients that underwent surgical treatment for NEC with vacuum-assisted closure (VAC) of the abdomen as compared with traditional laparotomy, bowel resection, and ostomy creation.

Methods A retrospective review identified 26 patients from 2007 to 2012 with NEC. Overall, 17 patients were treated with laparotomy, and 9 were treated with laparotomy and VAC (LapVac). Age, weight, preoperative and postoperative mean airway pressure, length of bowel resected, duration on total peripheral nutrition, time until initiation of feeds, and length of stay were assessed. A Student's t-test was used for statistical analysis.

Results Nine LapVac patients underwent a total of $1.2 \pm 1.3$ VAC changes and had open abdomens for $13.1 \pm 19.1$ days. LapVac and traditional laparotomy patients had similar outcomes with respect to amount of bowel resected, time on a ventilator, time to initiation of feeds, and length of hospital stay. Two of nine patients (22\%) in the LapVac group were placed in continuity without the need for an ostomy. We identified a subset of patients in the LapVac group that demonstrated signs of abdominal compartment syndrome (ACS), exhibiting mean airway pressures greater than $15 \mathrm{~cm} \mathrm{H}_{2} \mathrm{O}$ preoperatively. Patients with ACS treated with VAC therapy had shorter time to initiation of feeds $(p=0.047)$ and shorter lengths of stay $(p=0.0395)$ as compared with traditional laparotomy.

Conclusion Our data demonstrate that use of the wound VAC is a safe approach in the management of premature infants with NEC requiring surgical intervention with outcomes comparable to standard surgical management. Use of the wound VAC may allow the establishment of bowel continuity and abdominal closure without the need for an ostomy. VAC therapy may also hasten the recovery of NEC patients with concomitant ACS by eliminating the compartment syndrome. Larger studies are required to confirm this theory.
\end{abstract}




\section{Introduction}

Necrotizing enterocolitis (NEC) is the most common gastrointestinal emergency in the preterm infant and is characterized by variable damage to the intestinal tract, ranging from mucosal injury to full-thickness necrosis and perforation. ${ }^{1}$ The majority of NEC can be managed medically using gastric decompression, bowel rest, and intravenous antibiotics. Despite medical management, some patients develop fullthickness necrosis of the bowel wall and perforation, requiring operative intervention.

The standard surgical treatment for perforated NEC is laparotomy and resection of gangrenous or perforated bowel with the creation of an enterostomy and closure of the abdomen. ${ }^{2}$ Other options include resection with primary anastomosis, proximal diverting jejunostomy, and the "clip and drop" technique. ${ }^{3}$ When using the "clip and drop" technique, the bowel is resected without the creation of a stoma or anastomosis. ${ }^{4}$ Bowel with uncertain viability is retained during the first operation with a final decision made at a later time.

For the past decade, vacuum-assisted closure (VAC) has become an accepted modality for children and adults with complex wounds, abdominal wall defects, and damage control situations for critically ill patients. The wound VAC is an efficient and inexpensive way to temporarily close the abdomen. It provides sterile coverage of the abdominal contents, while allowing for reduction in intra-abdominal pressures, observation of fluid losses, decontamination of intra-abdominal spillage, and easy reentry into the abdominal cavity. Fenton et al concluded that VAC is a safe and effective temporary abdominal closure method in infants. ${ }^{5}$ The purpose of this study was to compare infants with NEC treated with the "clip and drop" technique and VAC placement with those infants treated with traditional bowel resection, ostomy creation, and immediate abdominal closure.

\section{Methods}

After the institutional review board approval a retrospective chart review of all patients with NEC from January 1, 2007 to December 31, 2012 at St Christopher's Hospital for Children was performed. Data collection included patient demographics, time to development and diagnosis of NEC, need for mechanical ventilation, time to initiation of feeds, time to goal feeds, length of hospital stay, and any complications. Analysis was conducted using a Student's $t$-test.

The use of the VAC system (VAC, Kinetic Concepts, Inc. $[\mathrm{KCI}]$, San Antonio, Texas, United States) was at the discretion of the operating surgeon. A fenestrated plastic sheet was placed over the peritoneal contents to protect the bowel. A polyurethane foam dressing was then placed over the plastic sheeting and adherent tape was placed over the sponge and connected to the VAC tubing to create suction. The VAC pressure was set to $25 \mathrm{~mm} \mathrm{Hg}$. The VAC was changed every 2 to 4 days depending on the clinical situation. The LapVac was used for varied lengths of time, depending on the clinical situation. The VAC system was removed and the abdomen was either closed with an ostomy, or closed after a bowel anastomosis based on the patient's clinical condition and the surgeon's judgment.

\section{Results}

A retrospective chart review identified 26 patients with NEC requiring operative intervention. Overall, 17 patients underwent traditional exploratory laparotomy, with intestinal resection, creation of an ostomy, and immediate abdominal closure (group 1) and 9 patients underwent exploratory laparotomy and bowel resection leaving the bowel clipped, but in discontinuity, followed by VAC-assisted closure (group 2). The average gestational age of the traditional group was $29.3 \pm 5.3$ weeks (range, 24-40 weeks) with a recorded birth weight of 1,389 $\pm 906 \mathrm{~g}$ (range, 606-3,720 g). The gestational age of the LapVac group was $27.3 \pm 4.6$ weeks (range, 23-38 weeks) with a recorded birth weight of $1,118 \pm 900 \mathrm{~g}$ (range, $401-3,390 \mathrm{~g})$. There was no significant difference in age or weight at initial operation between the two groups (see - Table 1).

Preoperative and postoperative vital signs were assessed to determine how ill patients were before surgery and how well patients tolerated the procedure. Preoperative heart rate, mean arterial pressure, $\mathrm{PaO}_{2}$, and urinary output were statistically similar in both the traditional laparotomy and LapVac groups. Postoperative vital signs, including heart rate, mean arterial pressure, $\mathrm{PaO}_{2}$, and urinary output were also the same between both the groups (see - Table 2). There was no difference between the two cohorts with respect to the volume of blood products transfused intraoperatively. Length of bowel removed in the initial index case and total

Table 1 Demographics of study groups

\begin{tabular}{|l|l|l|l|}
\hline Demographics & Group $1(N=17)$ & Group 2 $(N=9)$ & $p$-Value \\
\hline Male:female ratio & $12: 5$ & $5: 4$ & \\
\hline Gestational age (wks) & $29.3 \pm 5.3(24-40)$ & $27.3 \pm 4.6(23-38)$ & 0.35 \\
\hline Birth weight (g) & $1,389 \pm 906(606-3,720)$ & $1,118 \pm 900(401-3,390)$ & 0.47 \\
\hline Age at index operation (d) & $30.7 \pm 21.4(2-67)$ & $25 \pm 19.3(1-51)$ & 0.51 \\
\hline Weight at index operation $(\mathrm{g})$ & $1,783.8 \pm 778.2(900-3,700)$ & $1,717.8 \pm 1,093.8(920-4,500)$ & 0.86 \\
\hline
\end{tabular}


Table 2 Clinical parameters of study groups

\begin{tabular}{|l|l|l|l|}
\hline Clinical parameters & Group $1(N=17)$ & Group $2(N=9)$ & $p$-Value \\
\hline Preoperative heart rate $(\mathrm{bpm})$ & $165.9 \pm 18.8(133-214)$ & $167.4 \pm 13.3(150-194)$ & 0.83 \\
\hline Postoperative heart rate $(\mathrm{bpm})$ & $177.6 \pm 24.2(117-222)$ & $172 \pm 20.8(144-213)$ & 0.56 \\
\hline Preoperative mean arterial pressure $(\mathrm{mm} \mathrm{Hg})$ & $44.5 \pm 10.9(31-68)$ & $49.6 \pm 11.6(35-70)$ & 0.28 \\
\hline Postoperative mean arterial pressure $(\mathrm{mm} \mathrm{Hg})$ & $44.1 \pm 12.0(25-62)$ & $42.6 \pm 12.4(26-61)$ & 0.77 \\
\hline Preoperative mean airway pressure $\left(\mathrm{cm} \mathrm{H}_{2} \mathrm{O}\right)$ & $11.7 \pm 2.8(8-19)$ & $17.6 \pm 7.6(11-30)$ & 0.02 \\
\hline Postoperative mean airway pressure $\left(\mathrm{cm} \mathrm{H}_{2} \mathrm{O}\right)$ & $11.1 \pm 1.4(7-13)$ & $10.7 \pm 3.3(5-17)$ & 0.68 \\
\hline Blood products received $(\mathrm{mL})$ & $53.5 \pm 49.9(0-190)$ & $41.7 \pm 35.5(0-100)$ & 0.54 \\
\hline Length of bowel resected at index operation $(\mathrm{cm})$ & $21.6 \pm 17.2(3.5-55), n=16$ & $15.5 \pm 16.5(0-46)$ & 0.40 \\
\hline Total length of bowel resected $(\mathrm{cm})$ & $24.2 \pm 16.7(3.5-55), n=16$ & $22.0 \pm 16.7(7.1-50)$ & 0.75 \\
\hline Number of VAC changes & N/A & $1.2 \pm 1.3(0-3)$ & N/A \\
\hline Days until abdominal closure & N/A & $13.1 \pm 19.1(2-63)$ & N/A \\
\hline Days until bowel continuity established & $99.8 \pm 125.9(25-180), n=16$ & $66.3 \pm 39.6(2-122)$ & 0.45 \\
\hline
\end{tabular}

Abbreviations: bpm, beats per minute; N/A, not applicable; VAC, vacuum-assisted closure.

length of bowel removed for all surgeries were also statistically similar for both the groups.

There was a statistically significant difference in the two groups' mean airway pressures both preoperatively and postoperatively. Patients who received LapVac had a higher preoperative mean airway pressure $\left(17.6 \pm 7.6 \mathrm{~cm} \mathrm{H}_{2} \mathrm{O}\right)$ compared with those in the traditional laparotomy group $\left(11.7 \pm 2.8 \mathrm{~cm} \mathrm{H}_{2} \mathrm{O}\right.$; $p=0.0179$ ). Around 24 hours after the index surgery, the LapVac patients' mean airway pressure $\left(10.7 \pm 3.3 \mathrm{~cm} \mathrm{H}_{2} \mathrm{O}\right)$ returned to a value similar to those of the traditional group (11.1 $\left.\pm 1.4 \mathrm{~cm} \mathrm{H}_{2} \mathrm{O} ; p=0.67\right)$. On average, the LapVac patients took approximately 13.1 days until final abdominal closure.

Postoperative outcomes were similar for the traditional laparotomy group and the LapVac group. Both groups had similar total days on the ventilator, similar durations on total peripheral nutrition (TPN) or similar times until initiation of enteral feeds (see -Table 3). Ostomy complications were similar in both the groups. Overall 11 of 17 patients in the traditional laparotomy group had stoma complications, and 6 of 9 patients in the LapVac group had stoma complications. However, two of nine patients (22\%) in the LapVac group were placed back into continuity without the need for an ostomy, while no patients in the traditional group avoided an ostomy.

Upon review of the nine LapVac patients, we identified a subset group of patients that had significantly better out- comes than the rest of the study patients. These five patients had significantly higher mean airway pressures preoperatively $\left(23.8 \pm 5.8 \mathrm{~cm} \mathrm{H}_{2} \mathrm{O}\right)$ versus the traditional exploratory laparotomy patients $\left(11.7 \pm 2.8 \mathrm{~cm} \mathrm{H}_{2} \mathrm{O} ; p<0.0001\right)$. We considered this group (group 3 ) to have abdominal compartment syndrome (ACS) at their index operation (see - Table 4). Use of the wound VAC allowed normalization in their mean airway pressures over the 24 hours following their operation, returning their mean airway pressures to values similar to group 1 (see -Table 5). Comparing group 3 patients to the traditional laparotomy group, there was no statistical difference in blood transfusion volume, length of bowel removed in the index operation, or the total length of bowel removed. Nor was there a difference in total days on ventilator, or duration on TPN. However, group 3 patients had significantly shorter times until initiation of enteral feeds and significantly shorter lengths of stay as compared with the traditional laparotomy group (see - Table 6). One of the five patients in group 3 did not require an ostomy before abdominal closure.

\section{Discussion}

While often treated medically, NEC can become severe and require surgical intervention if intestinal perforation occurs or patients fail to respond to medical management. The

Table 3 Postoperative outcomes

\begin{tabular}{|l|l|l|l|}
\hline Outcome & Group $1(N=17)$ & Group 2 $(N=9)$ & $p$-Value \\
\hline Total days on ventilator & $44.1 \pm 37.6(4-111)$ & $45.8 \pm 25.6(10-85)$ & 0.90 \\
\hline Duration on TPN after closure (d) & $97.7 \pm 95.1(14-360)$ & $75.4 \pm 69.5(11-229)$ & 0.54 \\
\hline Time until initiation of enteral feeds (d) & $38.1 \pm 30.7(8-141)$ & $24.9 \pm 22.2(9-74)$ & 0.27 \\
\hline Length of hospital stay (d) & $173.1 \pm 110.3(25-386)$ & $179.6 \pm 55.3(84-2,450)$ & 0.87 \\
\hline Ostomy complications & 11 & 6 & 0.92 \\
\hline
\end{tabular}

Abbreviation: TPN, total peripheral nutrition. 
44 Vacuum-Assisted Closure for Managing Surgical NEC Sea et al.

Table 4 Demographics of traditional group versus LapVac patients with concomitant abdominal compartment syndrome

\begin{tabular}{|l|l|l|l|}
\hline Demographics & Group $1(N=17)$ & Group 3 $(N=5)$ & $p$-Value \\
\hline Male:female ratio & $12: 5$ & $3: 2$ & \\
\hline Gestational age (wks) & $29.3 \pm 5.3(24-40)$ & $28.6 \pm 5.8(23-38)$ & 0.80 \\
\hline Birth weight $(g)$ & $1,389 \pm 906(606-3,720)$ & $1,444 \pm 1,122(530-3,390)$ & 0.91 \\
\hline Age at index operation (d) & $30.7 \pm 21.4(2-67)$ & $30.8 \pm 16.0(10-47)$ & 0.99 \\
\hline Weight at index operation $(\mathrm{g})$ & $1,783.8 \pm 778.2(900-3,700)$ & $1,960 \pm 1,462(920-4,500)$ & 0.72 \\
\hline
\end{tabular}

Abbreviation: LapVac, treated with laparotomy and vacuum-assisted closure.

Table 5 Clinical parameters of traditional group versus LapVac patients with concomitant abdominal compartment syndrome

\begin{tabular}{|l|l|l|l|}
\hline Clinical parameters & Group $1(N=17)$ & Group $3(N=5)$ & $p$-Value \\
\hline Preoperative heart rate $(\mathrm{bpm})$ & $165.9 \pm 18.8(133-214)$ & $175.0 \pm 14.0(156-197)$ & 0.33 \\
\hline Postoperative heart rate $(\mathrm{bpm})$ & $177.6 \pm 24.2(117-222)$ & $180.0 \pm 24.0(158-213)$ & 0.85 \\
\hline Preoperative mean arterial pressure $(\mathrm{mm} \mathrm{Hg})$ & $44.5 \pm 10.9(31-68)$ & $46.2 \pm 9.1(38-60)$ & 0.76 \\
\hline Postoperative mean arterial pressure $(\mathrm{mm} \mathrm{Hg})$ & $44.1 \pm 12.0(25-62)$ & $48.2 \pm 10.6(39-61)$ & 0.50 \\
\hline Preoperative mean airway pressure $\left(\mathrm{cm} \mathrm{H} \mathrm{H}_{2} \mathrm{O}\right)$ & $11.7 \pm 2.8(8-19)$ & $23.8 \pm 5.8(16-30)$ & $<0.0001$ \\
\hline Postoperative mean airway pressure $\left(\mathrm{cm} \mathrm{H} \mathrm{H}_{2} \mathrm{O}\right)$ & $11.1 \pm 1.4(7-13)$ & $10.8 \pm 4.6(5-17)$ & 0.81 \\
\hline Blood products received $(\mathrm{mL})$ & $53.5 \pm 49.9(0-190)$ & $34.0 \pm 38.5(0-100)$ & 0.43 \\
\hline Length of bowel resected at index operation $(\mathrm{cm})$ & $21.6 \pm 17.2(3.5-55), n=16$ & $5.9 \pm 6.3(0-15)$ & 0.06 \\
\hline Total length of bowel resected $(\mathrm{cm})$ & $24.2 \pm 16.7(3.5-55), n=16$ & $13.9 \pm 8.2(7.1-24.3)$ & 0.20 \\
\hline Number of VAC changes & N/A & $1.0 \pm 1.4(0-3)$ & N/A \\
\hline Days until abdominal closure & N/A & $17.6 \pm 25.8(2-63)$ & N/A \\
\hline Days until bowel continuity established & $99.8 \pm 125.9(2-180), n=16$ & $70.2 \pm 41.2(12-122)$ & 0.62 \\
\hline
\end{tabular}

Abbreviations: bpm, beats per minute; LapVac, treated with laparotomy and VAC; N/A, not applicable; VAC, vacuum-assisted closure.

mainstay of treatment for surgical NEC is exploratory laparotomy with bowel resection, creation of an ostomy, and closure of the abdomen. However, our institution has been using abdominal wound VAC therapy as an alternate therapeutic option for infants requiring surgery for NEC. This is the first report to compare the use of VAC therapy in surgical NEC patients with those treated with traditional surgical principles for NEC.

We began using VAC therapy with the goal of allowing abdominal decontamination, while the infant was recovering from the massive systemic inflammatory response seen in infants with severe NEC. Second, we hoped to be able to reanastomose the infants' intestine without the need for an ostomy. Avoidance of an ostomy eliminates complications associated with an ostomy, such as dehydration from high ostomy output, skin breakdown, and ostomy prolapse or stenosis, and also obviates the need for a second operation to reanastomose the intestine. Our study shows the high rate of stoma complications in this patient population with 17 of the 26 (65\%) patients having either high ostomy output or ostomy stenosis. However, use of the wound VAC was successful in preventing the need for an ostomy in two of the nine

Table 6 Clinical outcomes of traditional group versus LapVac patients with concomitant abdominal compartment syndrome

\begin{tabular}{|l|l|l|l|}
\hline Outcomes & Group $1(N=17)$ & Group $3(N=5)$ & $p$-Value \\
\hline Total days on ventilator & $44.1 \pm 37.6(4-111)$ & $37.8 \pm 22.0(10-64)$ & 0.73 \\
\hline Duration on TPN after closure $(d)$ & $97.7 \pm 95.1(14-360)$ & $33.0 \pm 31.0(11-87)$ & 0.16 \\
\hline Time until initiation of enteral feeds (d) & $38.1 \pm 30.7(8-141)$ & $9.8 \pm 1.1(9-11)$ & 0.05 \\
\hline Length of hospital stay (d) & $173.1 \pm 110.3(25-386)$ & $148 \pm 53(84-201)$ & 0.04 \\
\hline Ostomy complications & 11 & 3 & 0.11 \\
\hline
\end{tabular}

Abbreviation: TPN, total peripheral nutrition. 
LapVac patients (22\%), while none of the traditional laparotomy patients avoided an ostomy.

When using the VAC following intestinal resection and clipping and dropping the intestine back into the abdomen, we noticed that the infants tolerated the postoperative period well. Our data show that in comparison to the standard laparotomy group, the LapVac group had statistically similar preoperative and postoperative vital signs, including heart rate, mean arterial pressure, $\mathrm{PaO}_{2}$, and urinary output. This indicates that neither group was more severely ill before surgery and that both the groups tolerated the surgical interventions similarly. Neither surgical intervention was superior in reduction of blood transfusions or preserving bowel length. Both the groups also had similar times on the ventilator, similar times until the initiation of feeds, and similar lengths of stay in the hospital. This data leads us to conclude that VAC therapy is an effective and safe option in the surgical management of NEC, and in some cases, may allow for reestablishment of bowel continuity without the need for an ostomy.

We identified a subset of five patients in the LapVac group that improved more rapidly than the rest of the study patients. These patients were judged to have ACS, due to their significantly elevated mean airway pressures compared with the rest of the patients in the study. Traditionally, ACS is determined by an elevation in bladder pressures. ${ }^{6}$ However, in the neonatal intensive care unit, where bladder pressures are rarely measured, elevated airway pressures are an established parameter for the diagnosis of ACS. ${ }^{7}$ These five patients had ACS based on elevated mean airway pressures of an average $23.6 \mathrm{~cm} \mathrm{H}_{2} \mathrm{O}$, which were statistically increased as compared with the rest of the patients in the study. Use of the wound VAC in this group reduced their postoperative mean airway pressures to a mean of $10.7 \mathrm{~cm}$ of $\mathrm{H}_{2} \mathrm{O}$, which was comparable to the rest of the patients.

It is known that ACS has deleterious effects on multiple organ systems, resulting in potential devastating patient outcomes. In this subset of patients the wound VAC eliminated the ACS. Subsequently, these five patients had a significantly shorter time to initiation of feeds and shorter lengths of stay when compared with patients treated with traditional laparotomy. We believe that by treating the ACS, the wound VAC improved this group's outcome. Admittedly, this is a small number of patients and more research is needed to confirm that the treatment of the ACS is the reason for the improvement in outcomes in patients with surgical NEC. However, the use of wound VAC therapy has been shown to be an effective treatment for ACS in the critically ill patients from many other intra-abdominal disease processes in both adults $^{8-11}$ and children, ${ }^{12-17}$ so it seems plausible to believe that it could work in infants with NEC.

In summary, our data demonstrate that use of the wound VAC is a safe approach in the management of premature infants with NEC requiring intervention with outcomes comparable to standard surgical management. In some cases, use of the wound VAC may allow the establishment of bowel continuity and abdominal closure without the need for an ostomy. VAC therapy may also hasten the recovery of NEC patients with concomitant ACS by eliminating the compartment syndrome. Larger studies are required to confirm this theory.

\section{Conflict of Interest}

None.

\section{References}

1 Tudehope DI. The epidemiology and pathogenesis of neonatal necrotizing enterocolitis. J Paediatr Child Health 2005;41(4):167-168

2 Henry MC, Moss RL. Neonatal necrotizing enterocolitis. Semin Pediatr Surg 2008;17(2):98-109

3 Pierro A. The surgical management of necrotising enterocolitis. Early Hum Dev 2005;81(1):79-85

4 Vaughan WG, Grosfeld JL, West K, Scherer LR III, Villamizar E, Rescorla FJ. Avoidance of stomas and delayed anastomosis for bowel necrosis: the 'clip and drop-back' technique. J Pediatr Surg 1996;31(4):542-545

5 Fenton SJ, Dodgion CM, Meyers RL, Nichol PF, Scaife ER. Temporary abdominal vacuum-packing closure in the neonatal intensive care unit. J Pediatr Surg 2007;42(6):957-960, discussion 960-961

6 Carlotti AP, Carvalho WB. Abdominal compartment syndrome: A review. Pediatr Crit Care Med 2009;10(1):115-120

7 Schierz IA, Giuffrè M, Piro E, et al. Predictive factors of abdominal compartment syndrome in neonatal age. Am J Perinatol 2014; 31(1):49-54

8 Batacchi S, Matano S, Nella A, et al. Vacuum-assisted closure device enhances recovery of critically ill patients following emergency surgical procedures. Crit Care 2009;13(6):R194

9 Amin AI, Shaikh IA. Topical negative pressure in managing severe peritonitis: a positive contribution? World J Gastroenterol 2009; 15(27):3394-3397

10 Bee TK, Croce MA, Magnotti LJ, et al. Temporary abdominal closure techniques: a prospective randomized trial comparing polyglactin 910 mesh and vacuum-assisted closure. J Trauma 2008;65(2): 337-342, discussion 342-344

11 Perez D, Wildi S, Demartines N, Bramkamp M, Koehler C, Clavien PA. Prospective evaluation of vacuum-assisted closure in abdominal compartment syndrome and severe abdominal sepsis. J Am Coll Surg 2007;205(4):586-592

12 Gutierrez IM, Gollin G. Negative pressure wound therapy for children with an open abdomen. Langenbecks Arch Surg 2012; 397(8):1353-1357

13 Lopez G, Clifton-Koeppel R, Emil S. Vacuum-assisted closure for complicated neonatal abdominal wounds. J Pediatr Surg 2008; 43(12):2202-2207

14 Caniano DA, Ruth B, Teich S. Wound management with vacuumassisted closure: experience in 51 pediatric patients. J Pediatr Surg 2005;40(1):128-132

15 McCord SS, Naik-Mathuria BJ, Murphy KM, et al. Negative pressure therapy is effective to manage a variety of wounds in infants and children. Wound Repair Regen 2007;15(3):296-301

16 Pauniaho SL, Costa J, Boken C, Turnock R, Baillie CT. Vacuum drainage in the management of complicated abdominal wound dehiscence in children. J Pediatr Surg 2009;44(9): 1736-1740

17 Kirkpatrick AW, Roberts DJ, De Waele J, et al; Pediatric Guidelines Sub-Committee for the World Society of the Abdominal Compartment Syndrome. Intra-abdominal hypertension and the abdominal compartment syndrome: updated consensus definitions and clinical practice guidelines from the World Society of the Abdominal Compartment Syndrome. Intensive Care Med 2013;39(7): 1190-1206 